\title{
The metabolism of proline, a stress substrate, modulates carcinogenic pathways
}

\author{
James M. Phang $\cdot$ Steven P. Donald • \\ Jui Pandhare · Yongmin Liu
}

Received: 4 February 2008/Accepted: 5 March 2008/Published online: 10 April 2008

(C) Springer-Verlag 2008

\begin{abstract}
The resurgence of interest in tumor metabolism has led investigators to emphasize the metabolism of proline as a "stress substrate" and to suggest this pathway as a potential anti-tumor target. Proline oxidase, a.k.a. proline dehydrogenase (POX/PRODH), catalyzes the first step in proline degradation and uses proline to generate ATP for survival or reactive oxygen species for programmed cell death. POX/PRODH is induced by p53 under genotoxic stress and initiates apoptosis by both mitochondrial and death receptor pathways. Furthermore, POX/PRODH is induced by PPAR $\gamma$ and its pharmacologic ligands, the thiazolidinediones. The anti-tumor effects of PPAR $\gamma$ may be critically dependent on POX/PRODH. In addition, it is upregulated by nutrient stress through the mTOR pathway to maintain ATP levels. We propose that proline is made available as a stress substrate by the degradation of collagen in the microenvironmental extracellular matrix by matrix metalloproteinases. In a manner analogous to autophagy, this proline-dependent process for bioenergetics from collagen in extracellular matrix can be designated "ecophagy".
\end{abstract}

The content of this publication does not necessarily reflect the views or policies of the Department of Health and Human Services, nor does mention of trade names, commercial products, or organizations imply endorsement by the US Government.

J. M. Phang $(\bowtie) \cdot$ S. P. Donald · J. Pandhare

Laboratory of Comparative Carcinogenesis, Center for Cancer

Research, Building 538, Room 115, NCI-Frederick, Frederick,

MD 21702, USA

e-mail: phang@mail.ncifcrf.gov

Y. Liu

Basic Research Program, SAIC-Frederick, NCI-Frederick, Frederick, MD 21702, USA
Keywords Proline oxidase - Proline dehydrogenase . mTOR $\cdot \operatorname{PPAR} \gamma \cdot$ Apoptosis $\cdot$ Bioenergetics

$\begin{array}{ll}\text { Abbreviations } \\ \text { mTOR } & \text { Mammalian target of rapamycin } \\ \text { NFAT } & \text { Nuclear factor of activated T-cells } \\ \text { P5C } & \Delta^{1} \text {-Pyrroline-5-carboxylic acid } \\ \text { POX } & \text { Proline oxidase } \\ \text { PPAR } \gamma & \begin{array}{l}\text { Peroxisome proliferator-activated receptor } \\ \text { gamma }\end{array} \\ \text { PRODH } & \begin{array}{l}\text { Proline dehydrogenase } \\ \text { ROS }\end{array} \\ \text { Reactive oxygen species } \\ \text { TZDs } & \text { Thiazolidinediones }\end{array}$

\section{Introduction}

The resurgence of interest in metabolism and bioenergetics has focused mainly on glucose and on the pathways for glycolysis and oxidative phosphorylation (Warburg 1930; Dang and Semenza 1999; Fox et al. 2005; Pan and Mak 2007). Alternative substrates are also important, since they can contribute to bioenergetics during nutrient stress. The metabolism of proline has features which are unique (Adams 1970; Phang 1985; Phang et al. 2001), and the understanding of these features may elucidate poorly understood regulatory mechanisms and contribute to the design of novel therapeutic regimens and molecular targets for human diseases.

Proline is the only secondary amino acid which is proteogenic and it makes unique contributions to the structure of proteins (Adams 1970; Phang 1985), in particular to the physical properties of tissues and maintenance of organ architecture (Dixit et al. 1977). 
More recently, investigators have pointed out that protein-protein recognition is based primarily on the structure provided by proline as a helix disruptor, e.g., in SH2 and WW domains ( $\mathrm{Lu}$ et al. 2003). On the other hand, the special features of proline metabolism are not as well known. With its alpha nitrogen contained within a pyrrolidine ring, proline cannot be metabolized by generic amino acid enzymes, i.e., racemases, aminotransferases, and decarboxylases. Instead, a special family of enzymes has evolved for proline metabolism (Adams and Frank 1980; Phang 1985), enzymes with special tissue and subcellular localization and mechanisms of regulation. With this metabolic paradigm distinct from that for other amino acids, proline can respond to special bioenergetic demands and/or regulatory mechanisms. The consequences of this response have been the topic of recent studies (Liu et al. 2005; Pandhare et al. 2006).

Although specialized metabolic and regulatory functions for proline have been recognized in a variety of animal and plant species, the mechanisms for these effects have not been clearly elucidated. Nevertheless, it is worth mentioning that proline has been considered as an osmoprotectant in bacteria and in plants and as a defense against oxidative stress (Phang 1985). Of special interest is the role of proline as a bioenergetic substrate for insects in their initiation of flight (Micheu et al. 2000; Gade and Auerswald 2002). Because of this requirement, certain insects also developed the ability to taste proline (Carter et al. 2006). Plants needing pollinators have high proline content in floral nectar (Carter et al. 2006). Thus, proline is the basis for an important co-evolution between plant and animal species.

\section{Historical background}

From the work of Elijah Adams and Harold Strecker during the late 1950s and early 1960s, the enzymes of proline metabolism were described and characterized (Adams 1970). The oxidized congener of proline, $\Delta^{1}$ pyrroline-5-carboxylic acid (P5C), is in tautomeric equilibrium with glutamic- $\gamma$-semialdehyde (GSA). P5C is at the center of intermediary metabolism (Phang 1985; Phang et al. 2001) as the obligate intermediate in the interconversions of glutamic acid and ornithine, and, in fact, is the carbon bridge linking these two major metabolic cycles. P5C can be converted to glutamate by P5C dehydrogenase, but the synthesis of P5C or GSA is by a separate protein which incorporates two activities, an ATP-dependent kinase and a NADPH-dependent reductase (see the article by C.A. Hu, this issue). The step from ornithine is a reversible reaction catalyzed by ornithine aminotransferase.

\section{The proline cycle}

We recognized that $\mathrm{P} 5 \mathrm{C}$ is both the immediate precursor of proline and also its degradative product. These reactions are catalyzed by distinct enzymes with their characteristic subcellular localizations. This relationship led us to formulate and characterize the proline cycle. The main participant in this cycle is proline oxidase, also known as proline dehydrogenase, an enzyme bound to mitochondrial inner membranes which donates electrons through an intervening flavine adenine dinucleotide into site II of the electron transport chain (Fig. 1) (Adams 1970; Adams and Frank 1980). Cytochrome $c$ is the electron acceptor, and subsequent transfer of these electrons supports the generation of ATP. More recently, we showed that the electrons from proline can reduce oxygen to yield superoxide (Liu et al. 2005) (see below). P5C produced from the oxidation (dehydrogenation) of proline, emerges from mitochondria and is converted back to proline by cytosolic P5C reductase with reduced pyridine nucleotide, either NADH or NADPH, as cofactors. The higher affinity for NADPH suggests that it is the preferred cofactor (Merrill et al. 1989). P5C reductase participates in a metabolic interlock with glucose-6-phosphate dehydrogenase of the pentose phosphate pathway (Phang et al. 1980) transferring reducing equivalents generated by the oxidation of glucose in the pentose phosphate pathway into mitochondria to generate ATP (Hagedorn and

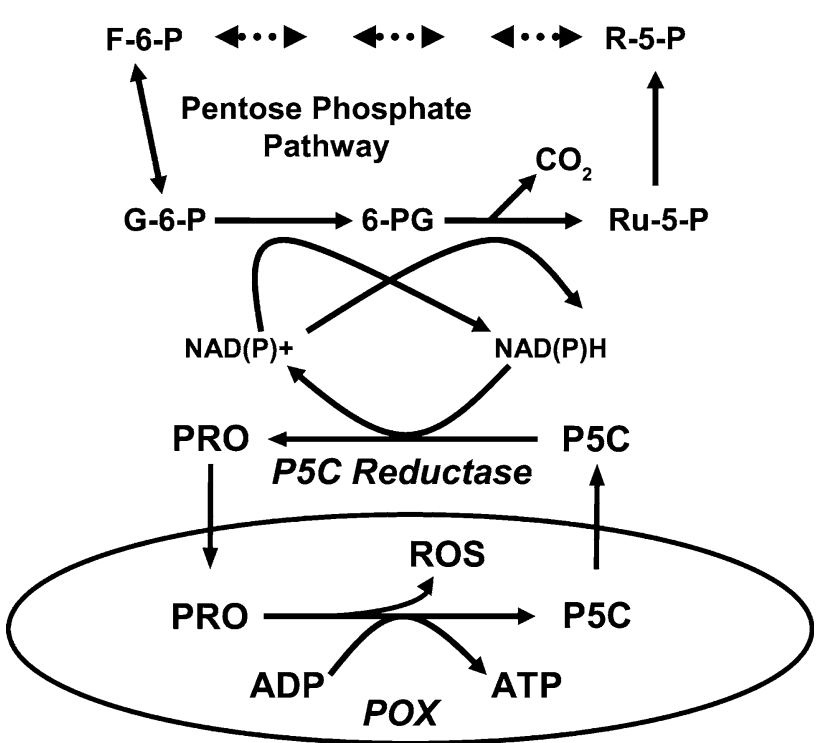

Fig. 1 The metabolic interlock between the proline cycle and pentose phosphate pathway. The proline cycle acts as a redox shuttle transferring reducing potential generated by the pentose phosphate shunt into mitochondria for the production of either ATP or ROS. Abbreviations for the pentose phosphate pathway: G-6-P glucose-6phosphate, 6- $P G$ 6-phosphogluconate, $R u-5-P$ ribulose-5-phosphate, $R$-5- $P$ ribose-5-phosphate, $F-6-P$ fructose-6-phosphate. Abbreviations for the proline cycle: $P O X$ proline oxidase, $R O S$ reactive oxygen species, $P R O$ proline, $P 5 C$ pyrroline-5-carboxylate 
Phang 1983; Hagedorn and Phang 1986). Although, this interconnection was recognized as novel, critics pointed out that the magnitude of bioenergy contributed by the cycling of proline is trivial compared to that from either tricarboxylic acid cycle or glycolytic pathway. Consequently, the proline cycle was assigned little physiologic relevance.

\section{POX/PRODH is p53-induced gene 6 (PIG6)}

In a seminal work, Polyak et al. (1997) used serial analysis of gene expression (SAGE) to identify the genes responding to $\mathrm{p} 53$, considered among the most important cancer suppressor proteins. Of 7202 genes monitored by this method, the overexpression of p53 induced only 14 genes greater than seven-fold, and these were designated as PIGs (p53induced genes). Proline oxidase/dehydrogenase was PIG6. This serendipitous finding caused considerable excitement for those of us working in the proline metabolism area, and stimulated the collaborative effort between our laboratory at the NCI-Frederick and the Valle laboratory at Johns Hopkins. C. Andy Hu had arrived as a postdoctoral fellow to work with David Valle in 1993 and had cloned several of the genes for proline-metabolizing enzymes (Phang et al. 2001). The gene for human proline oxidase (POX) also known as proline dehydrogenase (PRODH) was cloned in the Valle lab and the full-length cDNA sequence of human POX was submitted to Genebank in 1996. Subsequently in 1997, Campbell et al. (1997) identified POX as the human homologue of the Drosophila melanogaster sluggish-A (proline oxidase) gene that was mapped to 22q11.2. The cDNA sequence encoding the murine counterpart was published in 1999 by Gogos et al. (1999). We will refer to this enzyme as POX/PRODH and to the gene as PRODH. Jian $\mathrm{Yu}$ in the Vogelstein lab together with C. Andy $\mathrm{Hu}$ made a $P R O D H$ expression construct controlled by a tet-off promoter, and the overexpression of POX/PRODH was documented by enzyme activity and protein. Steve Donald and Xiao-Ya Sun soon showed that the overexpression of POX/PRODH was accompanied by the production of proline-dependent reactive oxygen species (Donald et al. 2001). Soon after, several laboratories independently demonstrated that overexpression of POX/PRODH-mediated proline-dependent apoptosis (Hu et al. 2001; Maxwell and Rivera 2003). The proline-dependent generation of ROS mediating the activation of the caspase cascade was shown by Liu et al. (2005) and Hu et al. (2007).

\section{POX/PRODH generates superoxide to initiate apoptosis}

Using co-transfections of SOD2, SOD1 (coding for $\mathrm{Mn}$ superoxide dismutase and CuZn superoxide dismutase, respectively), and $C A T$ (coding for catalase) individually or in combination, superimposed on the overexpression of POX/PRODH, Liu et al. (2005) showed that POX/PRODH generates mitochondrial superoxide, and it is this step which plays a critical role in initiating apoptosis. Apoptosis was confirmed using TUNEL assays, DNA laddering, and cell cytometry (Liu et al. 2005; Hu et al. 2007). Thus, it was convincingly demonstrated that POX/PRODH mediates the generation of mitochondrial superoxide and these apoptotic effects can be blocked by forced expression of SOD2 but not SOD1 or CAT. Thus, the intrinsic (mitochondrial) apoptosis pathway could be activated by POX/ PRODH activity downstream and independent of p53 (Fig. 2).

\section{POX/PRODH also upregulates the death receptor pathway}

An alternative apoptotic pathway is mediated by the socalled extrinsic or "death receptor" pathway (Liu et al. 2006). Ligands such as tumor necrosis factor (TNF), TNFrelated apoptosis inducing ligand (TRAIL) and Fas ligand can bind to their respective membrane receptors and through an adaptor molecule, the Fas-associated death domain (FADD) activate caspase 8 and downstream caspases. Liu et al. (2006) showed that overexpression of

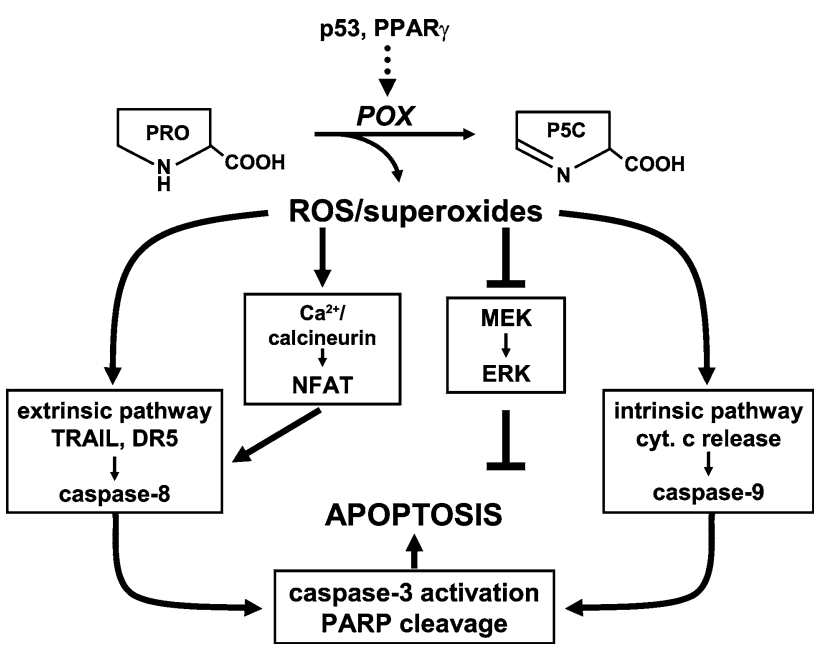

Fig. 2 Schematic of POX-mediated induction of apoptosis by both intrinsic (mitochondrial) and extrinsic (death receptor) pathways. $P O X$ is upregulated by stress signaling ( $p 53$ or $P P A R \gamma)$ which activates intrinsic pathway by generating superoxide and releasing cytochrome $c$ into the cytosol. NFAT (nuclear factor of activated $\mathrm{t}$-cells) is also activated by and POX induces TRAIL (tumor necrosis factor-related apoptosis inducing ligand) and DR5 (death receptor 5). Both limbs activate caspase 3 as seen by increased PARP (poly adenosylribose polymerase) cleavage. The $M E K$ (mitogen-activated protein kinase kinase) and $E R K$ (extracellular-regulated kinase) are downregulated by POX-generated ROS 
POX/PRODH also activates this pathway by upregulating the nuclear factor of activated T-cells (NFAT) which activates the promoter for TRAIL. The activation of NFAT by POX/PRODH overexpression had been described by Maxwell and Rivera (2003). Furthermore, POX/PRODH overexpression downregulated the phosphorylation of several MAPK components, specifically, MEK and ERK. The decreased phosphorylation can be partially reversed by co-expressing SOD2. Thus, ROS is the mediating mechanism not only for apoptosis but also for the downregulation of the MAP kinase pathway associated with apoptosis (Fig. 2). The translation of these tissue culture mechanistic studies to effects in animals and human tumors has been undertaken and the results are confirmatory and will be published elsewhere.

\section{POX/PRODH generates superoxide radicals autogenously}

Since POX/PRODH is induced by p53 and its activity plays a critical role in the generation of superoxide to induce programmed cell death, it is tempting to speculate that POX/PRODH is structurally adapted to perform this task. Although it can contribute proline-derived electrons to the electron transport chain, it would offer no advantage over other substrate sources such as succinate or NADH. The work from Tanner's laboratory may shed light on this issue. Using the monofunctional proline oxidase/proline dehydrogenase from Thermus thermophilus, White et al. (2007) demonstrated that the FAD at the active site is exposed to solvent oxygen. The recombinant enzyme can indeed generate superoxide autogenously. In addition, an adjacent alpha helix may have the structural flexibility to mask the solvent-access site and thereby switch off superoxide generation. If the mammalian enzyme is structurally similar, this model may provide an explanation for a controlled generation of superoxide versus ATP using proline-derived electrons. It is tempting to speculate that the aforementioned mechanism embodied in the intrinsic structure of the enzyme may offer a special source of oxidizing signals.

\section{PPAR $\gamma$ and its ligands induce POX/PRODH}

To discover additional mechanisms (Fig. 3) by which POX/PRODH can be regulated, Jui Pandhare together with Sandra Cooper developed a $P R O D H$ promoter luciferase reporter construct which was efficiently transfected into several colorectal cancer cell lines (Pandhare et al. 2006). Transcription factors were co-transfected to screen for activators of the $P R O D H$ promoter. They found a modest effect with c-Jun, c-Fos, and P-65 of NF-kB. However,

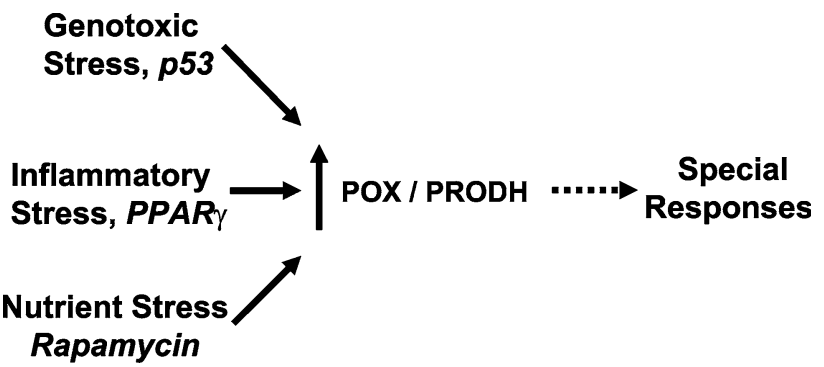

Fig. 3 Proline oxidase $(P O X / P R O D H)$ is upregulated by stress signaling. Genotoxic stress, p53; inflammatory stress, PPAR $\gamma$; and nutrient stress, rapamycin; all can upregulate POX to produce special cellular responses

none of these activated the $P R O D H$ promoter more than two-fold. Surprisingly, peroxisomal proliferator activated receptor gamma (PPAR $\gamma$ ) was the most potent activator of the PRODH promoter. Together with a PPAR $\gamma$ ligand, troglitazone, a thiazolidinedione (TZD), the activation was more than ten-fold. This was of interest because TZDs modulate several important metabolic systems. Clinicians have taken advantage of their effect on increasing insulin sensitivity to treat type 2 diabetes mellitus (Natali and Ferrannini 2006). In spite of recent concern over poorly understood side effects, these pharmacologic ligands of $\operatorname{PPAR} \gamma$ produce desirable therapeutic effects and have been widely used (Strauss and Glass 2007). Also interesting are their effects in decreasing the progression of atherosclerosis (Van Wijk and Rabelink 2005). Presumably, the mechanism is due to the inhibition of macrophages and their inflammatory signaling. Importantly, PPAR $\gamma$ and its ligands have effects on cancer (McAlpine et al. 2006; Li et al. 2006). Although there have been some contradictory data, the majority of studies using tissue culture systems have demonstrated that cancer cells treated with TZDs markedly decrease their growth or, in fact, undergo apoptosis (Hans and Roman 2007). Additionally, epidemiologic studies based on the population of patients with type 2 diabetes treated with TZDs suggest that the relative risk for lung cancer, but not colorectal cancer or prostate cancer, are significantly decreased with this drug (Govindarajan et al. 2007).

In luciferase reporter studies, Pandhare et al. (2006) found that the $P R O D H$ promoter is activated by co-transfection with PPAR $\gamma$ or by a treatment with several TZDs. The mechanism of this effect was shown to be transactivation, from electrophoretic mobility shift assays in which the peroxisomal proliferator response element (PPRE) of the $P R O D H$ promoter formed a complex with nuclear proteins and the quantity of this complex was increased by TZD and troglitazone. Additionally, the identity of the nuclear protein binding to PPRE was directly shown with chromosomal immunoprecipitation assays in which 
anti-PPAR $\gamma$ antibody was used to immunoprecipitate the cross-linked histone-DNA complex. In this complex, the PPRE sequence of the $P R O D H$ promoter was identified by polymerase chain reaction. Thus, $\operatorname{PPAR} \gamma$ and its ligands upregulate the expression of POX/PRODH through a transactivation of its promoter.

POX/PRODH appears to play a critical role in the apoptotic effects of PPAR $\gamma$. In normal medium conditions (DMEM, 10\% FBS, $2 \mathrm{mM}$ glutamine), the stimulation of ROS production by troglitazone in RKO (colorectal cancer) cells was markedly decreased by the knockdown of POX/PRODH expression with its anti-sense RNA (Pandhare et al. 2006). This important finding suggests that the anticancer effect of PPAR $\gamma$ may be critically dependent on a POX/PRODH induced, p53-independent, mechanism for activating apoptosis by both mitochondrial and death receptor pathways. Our finding in colorectal cancer cells was corroborated by workers using a cultured cell line derived from non-small cell lung cancer, the most common lung cancer. They showed that the apoptotic effects of pioglitazone, a commonly prescribed TZD, were completely blocked by knockdown of POX/PRODH with siRNA (Kim et al. 2007). Additional observations are required to generalize this very interesting effect linking PPAR $\gamma$ and apoptosis. Indeed, whether POX/PRODH plays a role in mediating the diverse effects of PPAR $\gamma$ ligands, including their effects on blood glucose in type 2 diabetes, is an important question.

\section{POX and bioenergetics}

Since PPAR $\gamma$ is involved in regulating the use of proline as metabolic substrate, we considered another regulatory pathway responding to the availability of substrates and nutrients, the mTOR pathway (Reiling and Sabatini 2006). This pathway integrates information from a number of metabolic sensors including: (1) growth factor signaling, (2) the availability of amino acids, and (3) the status of bioenergetics. Signaling from this pathway switches the cell from a biosynthetic, proliferating mode to non-proliferating survival mode. mTOR is a serine/ threonine kinase which phosphorylates initiation factors and ribosomal proteins to activate protein translation and cell proliferation, respectively. The downregulation of mTOR from substrate (glucose) deprivation was linked to upregulation of POX/PRODH. This upregulation helped to maintain cellular levels of ATP. The participation of $\mathrm{POX} / \mathrm{PRODH}$ in this bioenergetic response was corroborated by the maintenance of ATP levels that was blocked by POX/PRODH knockdown with siRNA or by inhibition of enzymatic activity with dehydroproline. This work was presented in an abstract (Pandhare et al.
2007; Pandhare et al. 2008) and will be published elsewhere.

The upregulation of POX/PRODH could channel carbons sequentially yield glutamate and $\alpha$-ketoglutarate, thereby supplying substrate for the tricarboxylic acid cycle. Since cancer cells primarily utilize glycolysis, the contribution of carbons is not routed into the TCA cycle. In fact, tissue culture cells primarily convert glutamine to lactate (Mazurek et al. 2001). On the other hand, increased POX/ PRODH would increase P5C and activate the proline cycle. Previous studies showed that the proline cycle participates in a metabolic interlock with the pentose phosphate pathway linked by NADPH/NADP ${ }^{+}$at the level of glucose-6phosphate dehydrogenase (G6PDH). The flux through G6PDH is regulated primarily by the availability of $\mathrm{NADP}^{+}$(Eggleston and Krebs 1974). In this context, the enzyme P5C reductase can be considered a P5C-dependent NADPH dehydrogenase, thereby linking the two pathways (Merrill et al. 1989).

\section{POX/PRODH and the pentose phosphate pathway}

To test whether the activation of POX/PRODH not only generated $\alpha$-ketoglutarate as substrate for the TCA cycle, but also activated the metabolism of glucose through the pentose phosphate pathway, we measured glucose metabolized either via the glycolytic pathway or via the pentose phosphate pathway in DLD-tet-off POX cells. To monitor glycolysis, we quantitated the production of ${ }^{3}\left[\mathrm{H}_{2} \mathrm{O}\right]$ from 5- ${ }^{3}[\mathrm{H}]$-glucose (Liedtke et al. 1992; Bauer et al. 2004) and for the pentose phosphate pathway, we recovered ${ }^{14}\left[\mathrm{CO}_{2}\right]$ production from $1-{ }^{14}[\mathrm{C}]$-glucose (Phang et al. 1980). In DLD-tet-off POX cells which overexpressed POX/PRODH when doxycycline is deleted from the medium, the increased POX/PRODH activity had little effect on glycolysis (data not shown). By contrast, the activity of the pentose phosphate pathway was increased more than fivefold when POX/PRODH was induced by the deletion of doxycycline, and an increase was seen over the entire range of glucose concentration (Fig. 4).

The aforementioned findings showed that the pentose phosphate pathway is linked with the proline cycle, but additional evidence was necessary to show that glucosederived reducing potential in the form of NADPH affected ATP levels via the cycling of proline. We first showed that the expression of POX/PRODH increased cellular ATP levels (Fig. 4), but this could have occurred by the contribution of proline to the TCA cycle. To test whether coupling with the pentose phosphate pathway was contributing to an increase in ATP, we treated cells with dehydroepiandrosterone (DHEA), a well-known inhibitor of G6PDH (Gordon et al. 1995), the rate-limiting enzyme 


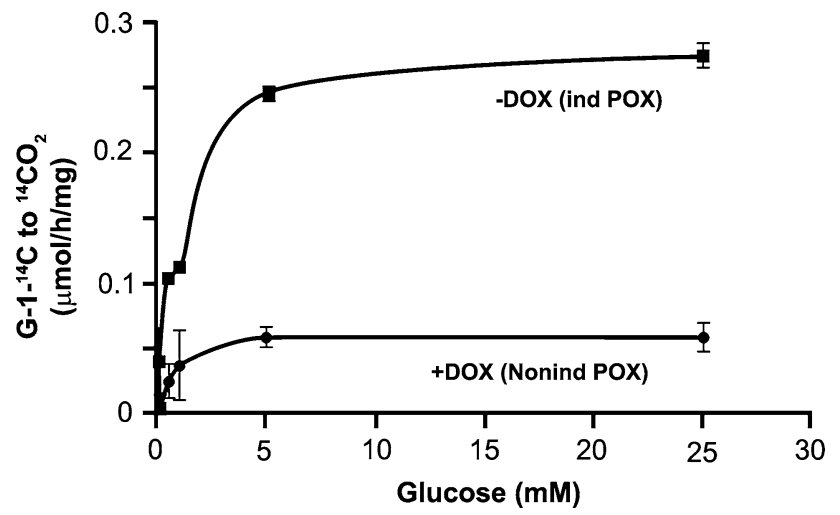

Fig. 4 The effect of POX induction on the pentose phosphate pathway. DLD-POX cells were cultured without doxycycline to induce POX. Control cells were cultured in the presence of doxycycline $\left(20 \mathrm{ng} \mathrm{ml}^{-1}\right)$. Cells cultured under both conditions were exposed to glucose- $1-{ }^{14}[\mathrm{C}]$ at concentrations of glucose shown and ${ }^{14}\left[\mathrm{CO}_{2}\right]$ collected by previously described methods (Phang et al. 1980). Activities are shown as micromoles $\mathrm{C}^{14}\left[\mathrm{O}_{2}\right]$ recovered from glucose- $1-{ }^{14}[\mathrm{C}] \mathrm{h}^{-1} \mathrm{mg}^{-1}$ cell protein

of the pentose phosphate pathway. We found that DHEA significantly decreased the POX-dependent increase in cellular ATP (Fig. 5). Thus, a significant amount of the increase in ATP is linked to the generation of NADPH by the pentose phosphate pathway.

\section{Collagen as a microenvironmental source of proline}

Although POX/PRODH can be activated by a variety of mechanisms, the availability of proline in the microenvironment of cells with upregulated POX/PRODH may be

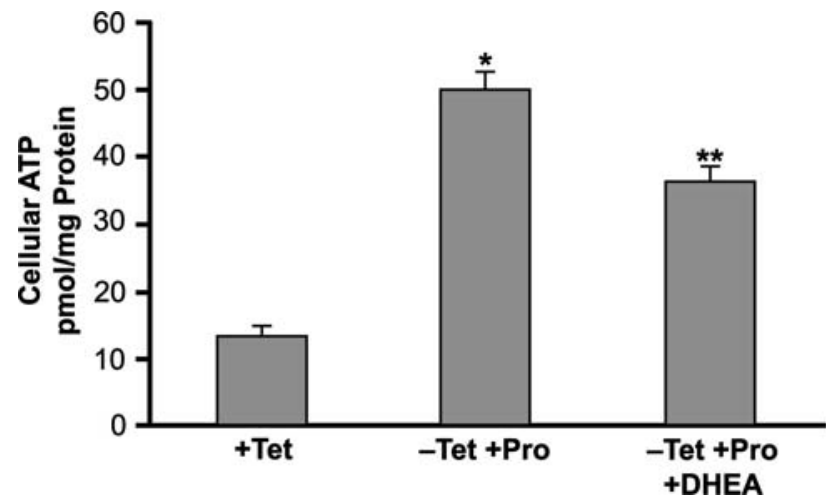

Fig. 5 Cellular ATP in DLD-POX cells. ATP was measured using luciferase method. ATP levels were higher when DOX was absent (POX-induced). The concentration of proline was $1 \mathrm{mM}$. However, the addition of DHEA (dehydro-epiandrosterone) at $100 \mu \mathrm{M}$ decreased ATP levels under these conditions. Final concentration of Dimethyl sulfoxide was $1 \%$ in control and treated preparations. Data represent mean and SD of at least three determinations. (*difference from control, + DOX, $P<0.001 ; * *$ difference from control, without DHEA, $P<0.01)$ limited because of compromise of the blood supply, e.g., with wound healing following tissue damage, or with tumor invasion (Laconi 2007). In this context, the degradation of collagen provides an alternative source. Proline and hydroxyproline are very abundant in collagen comprising $25 \%$ of the amino acid residues. Since collagen is also the most abundant protein in the body (by mass), there is a large reservoir of proline available (Dixit et al. 1977). In a $70 \mathrm{~kg}$ human, the total body protein is $11.0 \mathrm{~kg}$ and the total amount of proline and hydroxyproline can be calculated as $0.7 \mathrm{~kg}$ dry weight. Collagen makes up $80 \%$ of the proteins in the extracellular matrix and the activation of matrix metalloproteinases accompanying tumor invasion and during inflammation and wound healing is well known. Collagen is sequentially degraded first by MMPs and then by various proteases into large and small peptides and finally into imidodipeptides, dipeptides with proline or hydroxyoproline in the carboxyl terminus. The final release of proline or hydroxyproline is mediated by the activity of prolidase which furnishes proline as substrate for POX/ PRODH (Fig. 6).

Whether the degradation of collagen actually occurs under the conditions of inflammation and tumorigenesis has been addressed (Malemud 2006; Page-McCaw et al. 2007). In a model of inflammatory bowel disease, Fries et al. (1994) used trinitrobenzenesulphonic acid to induce colitis in rats. These animals showed a marked (greater than 2.5-fold) increase in their urinary excretion of hydroxyproline, an indicator of collagen degradation. Additionally, Reddy and Dhar (1989) produced an inflammatory arthritis in rats using injections of Freund's adjuvant. These animals also increased more than two-fold
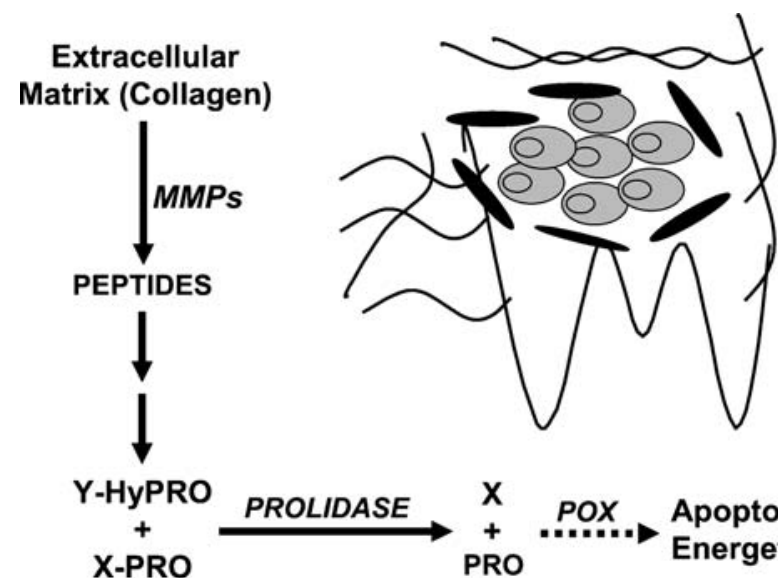

Fig. 6 Schematic of "Ecophagy". Tumor cells together with stromal cells activate matrix metalloproteinases to degrade collagen and other proteins in extracellular matrix. Sequential cleavage of peptides by proteases and peptidases terminate in imidodipeptides, i.e., dipeptides with proline or hydroxyproline at the carboxyl terminus. Prolidase releases proline (and hydroxyproline) as substrate for $P O X$ 
their excretion of hydroxyproline. Thus, accompanying induction of inflammation with either colitis or arthritis, the degradation of collagen was markedly increased.

Of special interest is the finding described by Marian and Mazzucco (1985) in their skin tumorigenesis model. In this model, shaved mice were painted first with an initiation chemical, e.g., dimethylbenzanthracene, followed by twice weekly paintings with a tumor promoter, i.e., 12-Otetradecanoyl-phorbol-13-acetate. Analysis of the dermal collagen showed that there was a marked degradation of collagen. Dermal hydroxyproline content decreased beginning at 2 weeks and plateaued throughout the 15 week study at a level almost $30 \%$ less than that of controls. Thus, the so-called tumor promoter which activated the "inflammatory paradigm" included a potent collagenolytic mechanism (Fig. 7).

\section{MMPs and proline}

A considerable body of studies have emphasized the family of metallo-enzymes involved in degrading collagen and other collagen-like structural proteins (Parks et al. 2004). Designated as matrix metalloproteinases (MMPs), their function, regulation, and modulation of cell behavior have been widely studied, especially as a component of inflammation and carcinogenesis (Malemud 2006; Stallings-Mann and Radisky 2007). These studies led to the proposal that MMPs may be a promising target for the prevention or treatment of cancer (Wang et al. 1994). But

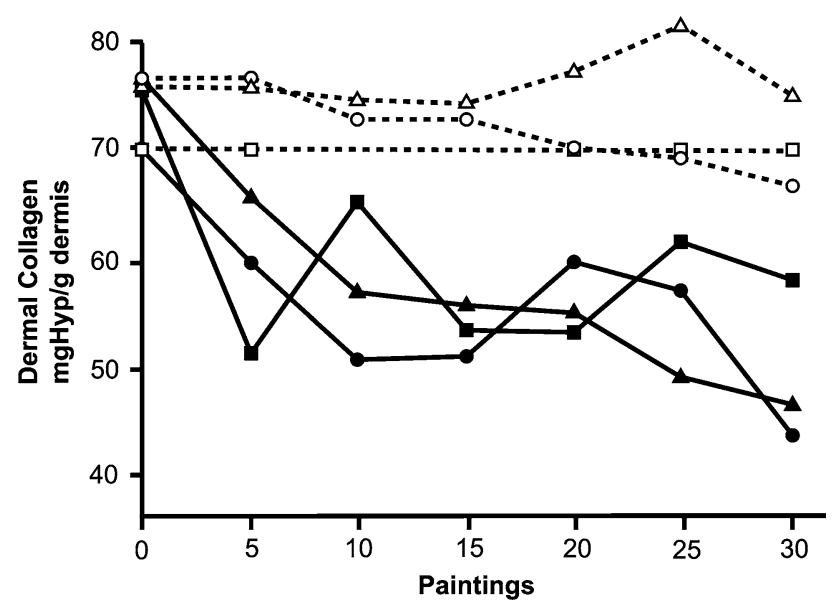

Fig. 7 Data from skin tumor model showing degradation of dermal collagen (adapted from Marian and Mazzucco 1985). Dermal collagen content is shown as microgram hydroxyproline per gram of dermis. Abscissa depicts number of paintings, i.e., topical applications (two per week) with 12-O-tetradecanoylphorbol-13acetate. Open figures represent control animals, and closed figures represent treated animals the clinical trials with broad-spectrum MMP inhibitors were generally disappointing and dampened the enthusiasm for targeting MMPs (Wagenaar-Miller et al. 2004). However, the discovery of the multiplicity of MMPs with their wide range of target substrates, and the finding that they participate in multiple processes, i.e., cell proliferation, differentiation, angiogenesis, and apoptosis, etc., have led to a resurgence of interest and research effort (PageMcCaw et al. 2007).

To explain these effects of MMPs, investigators have focused on the selective and limited cleavage of specific substrates including growth factors and their receptors thereby activating or releasing them to modify cell behavior. Although, the degradation of collagens into free amino acids and peptides is supported convincingly by the aforementioned studies, their release of substrates for metabolism, i.e., proline and hydroxyproline, has not been considered. With the accumulating evidence that proline oxidase is upregulated by p53 (Polyak et al. 1997; Donald et al. 2001; Hu et al. 2001; Maxwell and Rivera 2003; Hu et al. 2007), by PPAR $\gamma$, and its ligands (Pandhare et al. 2006; Kim et al. 2007) and by rapamycin and AICAR (Pandhare et al. 2007) (Fig. 3), the possibility of a metabolic endpoint for this regulation must be seriously considered. Additionally, an endpoint for the regulation of MMPs in cancer, arthritis, and cardiovascular disease (Malemud 2006) may be to supply proline and hydroxyproline as stress substrates.

\section{Prolidase and collagen degradation}

An important link in the mobilization of proline from the degradation of collagen by MMPs is the enzyme prolidase which catalyzes an obligate step to release C-terminal proline or hydroxyproline from imidodipeptides. An important lead was provided by the inherited disorder of prolidase deficiency. In these patients, a prominent feature of the clinical phenotype is lower extremity ulcers due to poor wound healing (Hechtman 2001; Lupi et al. 2006). Postmortem histology of both ulcerated areas as well as central organs, e.g., kidneys, revealed microangiopathy and atretic capillaries. These previously reported studies and our recent finding that proline from ECM degradation is used as substrate under conditions of nutrient stress prompted us to consider whether prolidase activity would generate angiogenic signaling (Semenza 2007). To answer this question, we made a prolidase expression construct and obtained stable transfectants with prolidase activities greater than ten-fold those of vector controls (Surazynski et al. 2007). In cells overexpressing prolidase, VEGF and Glut-1, two target genes of HIF-1, were upregulated, and elevated levels of HIF- $1 \alpha$ were demonstrated. The 
mechanism for the accumulation of HIF- $1 \alpha$ was due to the inhibition of VHL-dependent degradation. Whether the products of prolidase or subsequent metabolic products were inhibitors of HIF- $1 \alpha$ prolylhydroxylase activity has yet to be determined. Nevertheless, this signaling linkage, like that of hypoxia, suggests that the metabolic system senses ECM degradation as a stress condition which requires neoangiogenesis.

\section{Hydroxyproline degradation}

Although the focus of this review is on proline metabolism, the metabolism of hydroxyproline deserves mention. Unlike proline, preformed hydroxyproline is not proteogenic. Instead, proline is hydroxylated after peptide linkage (Adams 1970). In spite of these marked differences in proteogenic utilization, degradative metabolism of hydroxyproline, either from the diet or from protein turnover, parallels that for proline. The first degradative step, however, is catalyzed by distinct oxidases (dehydrogenases) encoded by different genes (Adams 1970; Adams and Frank 1980; Phang 1985). The second step, the degradation of P5C and OH-P5C, is catalyzed by a common enzyme, P5C dehydrogenase (Valle et al. 1979). Interestingly, $\mathrm{OH}-\mathrm{P} 5 \mathrm{C}$ is also a substrate for P5C reductase converting OH-P5C back to OH-PRO (Adams and Goldstone 1960). The recyling of $\mathrm{P} 5 \mathrm{C}$ to proline is not only the final step for de novo biosynthesis, but also recycles P5C to proline for incorporation into protein. In contrast, OH-PRO recycled from OH-P5C is not proteogenic. Adams and Goldstone (1960) thought it remarkable that OH-P5C could be utilized as substrate by P5C reductase with the generation of non-proteogenic OH-PRO and earmarked this an aspect of OH-PRO metabolism which required elucidation. Recently, Cooper et al. (2008) found that OH-POX encoded by $P R O D H 2$ was regulated by mechanisms similar to those for POX/PRODH in that both are induced by p53 and $\operatorname{PPAR} \gamma$. The induction of OH-POX by 553 in the presence of added medium OH-PRO generated ROS and initiated apoptosis. Thus, it appears that OH-POX is a redundant system which can back up the POX/PRODH apoptotic pathway. The cycling of OH-P5C to OH-PRO by P5C reductase comprises a hydroxyproline cycle which has no function other than for redox transfer and bioenergetics.

\section{Autophagy and ecophagy}

The utilization of proline as a microenvironmental stress substrate bears striking parallels to the process in which cellular materials are degraded as a source of bioenergy for either survival or programmed cell death. (Schwartz et al.
1993; Yorimatsu and Klonskyi 2005; Rubinsztein et al. 2005). Autophagy is the term used to denote this process. In this context, the utilization of proline (and hydroxyproline) that we have described may be designated "ecophagy" (Greek: oikos, home; phagein, to eat). Before a cell resorts to autophagy, i.e., consumes its own cellular components to maintain bioenergy and vital processes, it can activate ecophagy to mobilize constituents of its cellular microenvironment (its home) without compromising critical cellular functions.

In summary, since the proline cycle was formulated some 25 years ago, when we proposed that the metabolism of proline played a special role in redox transfers and bioenergetics, a body of persuasive evidence has accumulated defining the metabolic niche for its function. The screening for p53 gene targets revealed that POX/PRODH was encoded by a p53-induced gene (PIG6) and led to the elucidation of its role in intrinsic and extrinsic apoptosis. This effect was initiated by the generation of proline-dependent superoxides. In addition to the response to genotoxic stress (p53), POX/PRODH is also upregulated by PPAR $\gamma$ and its pharmacologic ligands, the thiazolidinediones. Importantly, the thiazolidinedione-mediated ROS-generation and apoptosis could be blocked by knockdown of POX/PRODH by siRNA, a finding suggesting that the anti-cancer effects of PPAR $\gamma$ and its ligands, at least in cultured cells, are critically dependent upon POX/PRODH. Finally, POX/PRODH is also upregulated under conditions of nutrient stress and the mTOR signaling system provides the mechanism. Thus, proline metabolized by POX/PRODH serves as a microenvironmentally derived stress substrate which can be used either for survival or for programmed cell death. This sequential process beginning with activation of MMPs and degradation of the collagen contained in ECM to furnish proline for POX may be designated, "ecophagy".

Acknowledgments This research is supported by the Intramural Research Program of the NIH, National Cancer Institute, Center for Cancer Research. This project also has been funded in part with Federal funds from the National Cancer Institute, National Institutes of Health under Contract No. N01-CO-12400.

\section{References}

Adams E (1970) Metabolism of proline and hydroxyproline. Int Rev Connect Tissue Res 5:1-91

Adams E, Frank L (1980) Metabolism of proline and the hydroxyprolines. Annu Rev Biochem 49:1005-1061

Adams E, Goldstone A (1960) Hydroxyproline metabolism. III. enzymatic synthesis of hydroxyproline from delta 1-pyrroline-3hydroxy-5-carboxylate. J Biol Chem 235:3499-3503

Bauer DE, Harris MH, Plas DR, Lum JJ, Hammerman POS, Rathmell JC, Riley JL, Thompson CB (2004) Cytokine stimulation of aeroic glycolysis in hematopoietic cells exceeds proliferative demand. FASEB J 18(11):1303-1305 10.10-96/fj03-1001 fje 
Campbell HD, Webb GC, Young JG (1997) A human homologue of the Drosophila melanogaster sluggish-A (proline oxidase) gene maps to $22 \mathrm{q} 11.2$, and is a candidate gene for type-I hyyperprolinemia. Hum Genet 101:69-74

Carter C, Shafir S, Yehonetaqn L, Palmer RG, Thornburg (2006) A novel role for proline in plant floral nectars. Naturwissenschaften 90:72-79

Cooper SK, Pandhare J, Donald SP, Phang JM (2008) A novel function for hydroxyproline oxidase in apoptosis through generation of reactive oxygen species. J Biol Chem (in press) Epub Feb 19

Dang CV, Semenza GL (1999) Oncogenic alterations of metabolism. Trends Biochem Sci 24:68-72

Dixit SN, Seyer JM, Kang AH (1977) Covalent structure of collagen: amino-acid sequence of chymotryptic peptides from the carboxyl-terminal region of $\alpha 2-\mathrm{CB} 3$ of chick-skin collagen. Eur $\mathrm{J}$ Biochem 81:599-607

Donald SP, Sun XY, Hu CA, Yu J, Mei JM, Valle D, Phang JM (2001) Proline oxidase, encoded by p53-induced gene-6, catalyzes the generation of proline-dependent reactive oxygen species. Cancer Res 61:1810-1815

Eggleston LV, Krebs HA (1974) Regulation of the pentose phosphate cycle. Biochem J 138:425-435

Fox CJ, Hammerman PS, Thompson CB (2005) Fuel feeds function: energy metabolism and the T-cell response. Nat Rev Immunol $5: 844-852$

Fries W, Giacomin D, Plebani M, Martin A (1994) Effect of experimental colitis on bone metabolism in the rat. Digestion 55:229-233

Gade G, Auerswald L (2002) Beetle's choice-proline for energy output: control by AKHS. Comp Biochem Physiol B Biochem Mol Biol 132:117-129

Gogos JA, Santha M, Takacs Z, Berk KD, Laine V, Luers LR, Nadler JV, Karayiorgou M (1999) The gene encoding proline dehydrogenase modulates sensorimotor gating in mice. Nat Genet 21:434-439

Gordon G, Mackow MC, Levy HR (1995) On the mechanism of interaction of steroids with human glucose-6-phosphate dehydrogenase. Arch Biochem Biophys 318:25-29

Govindarajan R, Ratnasinghe L, Simmons DL, Siegel ER, Midathada MV, Kim L, Kim PJ, Owens RJ, Lang NP (2007) Thiazolidinediones and the risk of lung, prostate, and colon cancer in patients with diabetes. J Clin Oncol 20:1461-1462

Hagedorn CH, Phang JM (1983) Transfer of reducing equivalents into mitochondria by the interconversions of proline and $\Delta^{1}$-pyrroline-5-carboxylate. Arch Biochem Biophys 225:95-101

Hagedorn CH, Phang JM (1986) Catalytic transfer of hydride ions from NADPH to oxygen by the interconversions of proline and delta 1-pyrroline-5-carboxylate. Arch Biochem Biophys 248:166-174

Han S, Roman J (2007) Peroxisome proliferator-activated receptor gamma: a novel target for cancer therapeutics? Anticancer Drugs 18:237-244

Hechtman P (2001) Prolidase deficiency. In: Scriver CR, Beaudet AL, Sly WS, Valle D (eds) Metabolic and molecular basis of inherited disease. McGraw-Hill, New York, pp 1839-1856

Hu C-AA, Yu J, Lin W-W, Donald SP, Sun X-Y, Almashanu S, Steel G, Phang JM, Vogelstein B, Valle D (2001) Overexpression of proline oxidase, a p53 induced gene (PIG6) induces reactive oxygen species generation and apoptosis in cancer cells. Proc Am Assoc Cancer Res 42:225

Hu C-AA, Donald SP, Yu J, Lin W-W, Liu Z, Steel G, Obie C Valle D, Phang JM (2007) Overexpression of proline oxidase induces proline-dependent and mitochondria-mediated apoptosis. Mol Cell Biochem 295:85-92

Kim KY, Ahn JH, Cheon HG (2007) Apoptotic action of peroxisome proliferator-activated receptor-gamma activation in human non small-cell lung cancer is mediated via proline oxidase-induced reactive oxygen species formation. Mol Pharmacol 72:674-685 (Epub 2007 May 29)

Laconi E (2007) The evolving concept of tumor microenvironments. Bioessays 29:738-744

Li MY, Lee TW, Yim AP, Chen GG (2006) Function of PPARgamma and its ligands in lung cancer. Crit Rev Clin Lab Sci 43:183-202

Liedtke AJ, Renstrom B, Nellis SH (1992) Correlation between [5$3 \mathrm{H}]$ glucose and [U-14C]deoxyglucose as markers of glycolysis in reperfused myocardium. Circ Res 71:689-700

Liu Y, Borchert GL, Donald SP, Surazynski A, Hu C-A, Weydert CJ, Oberley LW, Phang JM (2005) MnSOD inhibits proline oxidaseinduced apoptosis in colorectal cancer cells. Carcinogenesis 26:1335-1342

Liu Y, Borchert GL, Surazynski A, Hu C-A, Phang JM (2006) Proline oxidase activates both intrinsic and extrinsic pathways for apoptosis: the role of ROS/superoxides, NFAT and MEK/ERK signaling. Oncogene 25:5640-5647

Lu KP, Liou YC, Vincent I (2003) Proline-directed phosphorylation and isomerization in mitotic regulation and in Alzheimer's disease. Bioessays 25:174 181

Lupi A, Roossi A, Campari E, Pecora F, Lund AM, Elcioglu NH, Gultepe M, DiRocco M, Cetta G, Forlino A (2006) Molecular characterization of 6 patients with prolidase deficiency: identification of the first small duplication in the prolidase gene and of a mutation generating symptomatic and asymptomatic outcomes. J Med Genet 43:e58

Malemud DJ (2006) Matrix metalloproteinases (MMPs) in health and disease. Front Biosci 11:1696-2701

Marian B, Mazzucco K (1985) Dermal collagen metabolism during tumor promotion with 12 - $O$-tetradecanoylphorbol-13-acetate in mouse skin. Carcinogenesis 6:501-504

Maxwell SA, Rivera A (2003) Proline oxidase induces apoptosis in tumor cells, and its expression is frequently absent or reduced in renal carcinomas. J Biol Chem 278:9784-9789

Mazurek S, Zwerschke W, Jansen-Durr P, Eigenbrodt E (2001) Metabolic coooperation between different oncogenes during cell transformation: interaction between activated ras and HPV-16 E7. Oncogene 20:6891-6898

McAlpine CA, Barak Y, Matise I, Cormier RT (2006) Intestinalspecific PPARgamma deficiency enhances tumorigenesis in ApcMin/+ mice. Int J Cancer 119:2339-2346

Merrill MJ, Yeh GC, Phang JM (1989) Purified human erythrocyte pyrroline-5-carboxylate reductase. Preferential oxidation of NADPH. J Biol Chem 264:9352-9358

Micheu S, Crailsheim K, Leonhared B (2000) Importance of proline and other amino acids during honeybee flight. Amino acids 18:157-175

Natali A, Ferrannini E (2006) Effects of metformin and thiazolidinediones on suppression of hepatic glucose production and stimulation of glucose uptake in type 2 diabetes: a systematic review. Diabetologia 49:434-441 (Epub 2006 Feb 14)

Page-McCaw A, Ewald AJ, Werb Z (2007) Matrix metalloproteinases and the regulation of tissue remodelling. Nat Rev Mol Cell Biol 8:221-233

Pan JG, Mak TW (2007) Metabolic targeting as an anticancer strategy: dawn of a new era? Sci STKE 2007(381):pe14

Pandhare J, Cooper SK, Phang JM (2006) Proline oxidase, a proapoptotic gene, is induced by troglitazone: evidence for both peroxisome proliferator-activated receptor gamma-dependent and -independent mechanisms. J Biol Chem 281:2044-2052. (Epub 2005 Nov 21)

Pandhare J, Cooper SK, Donald SP, Phang JM (2007) The importance of proline metabolism under conditions of nutrient stress. Proc Am Assoc Cancer Res 48:4501 
Pandhare J, Cooper SK, Donald SP, Phang JM (2008) Significance of the proline metabolic pathway in the adaptation of cancer cells to nutrient/energy limitation (submitted)

Parks WC, Wilson CL, Lopez-Boado YS (2004) Matrix metalloproteinases as modulators of inflammation and innate immunity. Nat Rev Immunol 4:617-629

Phang JM (1985) The regulatory functions of proline and pyrroline-5carboxylic acid. Curr Topics Cell Regul 25:91-132

Phang JM, Downing SJ, Yeh GC (1980) Linkage of the HMP pathway to ATP generation by the proline cycle. Biochem Biophys Res Commun 93:462-470

Phang JM, Hu CA, Valle D (2001) Disorders of proline and hydroxyproline metabolism. In: Scriver CR, Beaudet AL, Sly WS, Valle D (eds) Metabolic and molecular basis of inherited disease. McGraw-Hill, New York, pp 1821-1838

Polyak K, Xia Y, Zweier JL, Kinzler KW, Vogelstein B (1997) A model for p53-induced apoptosis. Nature 389:300-305

Reddy GK, Dhar SC (1989) Metabolism of collagen in bone of adjuvant induced arthritic rat. Bone 10:439-445

Reiling JH, Sabatini DM (2006) Stress and mTOR signaling. Oncogene 25:6373-6383

Rubinsztein DC, DiFiglia M, Heinz N, Nixon RA, Qin ZH, Ravikumar B, Stefanes L, Tedkovsky A (2005) Autophagy and its possible roles in nervous system diseases, damage and repair. Autophagy 1:11-22

Semenza GL (2007) Hypoxia-inducible factor 1 (HIF-1) pathway. Sci STKE 2007(407):cm8

Schwartz LM, Smith SW, Jones ME, Osborne BA (1993) Do all programmed cell deaths occur via apoptosis? Proc Natl Acad Sci USA 90:980-984
Stallings-Mann M, Radisky D (2007) Matrix metalloproteinaseinduced malignancy in mammary epithelial cells. Cells Tissues Organs 185:104-110

Strauss DS, Glass CK (2007) Anti-inflammatory actions of PPAR ligands: new insights on cellular and molecular mechanisms. Trends Immunol 28:551-558. (Epub 2007 Nov 5)

Surazynski A, Donald SP, Cooper SK, Whiteside MA, Salnikow K, Liu Yk Phang JM (2007) Extracellular matrix and HIF-1 signaling: the role of prolidase. Int J Cancer Nov 12 (Epub ahead of print)

Valle D, Goodman SI, Harris SC Phang JM (1979) Genetic evidence for a common enzyme catalyzing dthe 2nd step in the degradation of proline and hydroxyproline. J Clin Invest 64:1365-1370

Van Wijk JP, Rabelink TJ (2005) Impact of thiazolidinedione therapy on atherogenesis. Curr Atheroscler Rep 7:369-374

Wagenaar-Miller RA, Gorden L, Matrisian LM (2004) Matrix metalloproteinases in colorectal cancer: is it worth talking about? Cancer Met Rev 23:119-135

Wang X, Fu X, Brown PD, Crimmin MJ, Hoffman RM (1994) Matrix metalloproteinase inhibitor BB-94 (batimastat) inhibits human colon tumor growth and spread in a patient-like orthotopic model in nude mice. Cancer Res 54:4726-4728

Warburg O (1930) Ueber den Stoffwechsel der Tumoren. Constable, London

White TA, Krishnan N, Becker DF, Tanner JJ (2007) Structure and kinetics of monofunctional proline dehydrogenase from Thermus thermophilus. J Biol Chem 282:14316-14327 (Epub 2007 Mar 7)

Yorimatsu T, Klonskhy DJ (2005) Autophagy, molecular machinery for self-eating. Cell Death Differ 12:1542-1552 